\title{
Nota BiogrÁficA
}

\section{Reseña de Alfredo Hipólito Llaña Garín (1913-1971): Primer ficólogo marino chileno}

\author{
Review of Alfredo Hipólito Llaña Garín (1913-1971): First Chilean marine phycologist
}

\author{
Vivian Montecino ${ }^{1,2^{*}}$, Nibaldo Bahamonde ${ }^{1}$ y Julio A. Vásquez ${ }^{(13}$ \\ ${ }^{1}$ Departamento de Ciencias Ecológicas, Facultad de Ciencias, Universidad de Chile, Las Palmeras 3425, Santiago, Chile \\ ${ }^{2}$ Instituto de Ecología y Biodiversidad (IEB), Universidad de Chile, Santiago, Chile \\ ${ }^{3}$ Departamento de Biología Marina, Facultad de Ciencias del Mar, Centro de Investigación y Desarrollo Tecnológico en Algas (CIDTA), \\ Universidad Católica del Norte Larrondo 1281, Coquimbo, Chile \\ *Autor corresponsal: vivianmontecino@uchile.cl
}

\begin{abstract}
Alfredo Llaña Garín, State Professor in Biological Sciences and Chemistry at the University of Chile (1937), is the forerunner of the study of marine algae in Chile. This review recalls his beginnings, his pioneering research in botany and work on microalgae in limnic waters, his contribution to the development of the Montemar Marine Biology Station, University of Chile and for 20 years at the University of Panama. Prof. Llaña also studies marine macroalgae, strengthening the importance of their commercial value. At present, Chile is recognized as one of the most important suppliers of seaweed for the extraction of agar, carrageenans and alginates, essential for the food, cosmetic, and biomedical industries.
\end{abstract}

Key words: Chilean botanical forerunner, micro and macroalgae

Resumen.- Alfredo Llaña Garín, Profesor de Estado en Ciencias Biológicas y Química de la Universidad de Chile (1937), es el precursor del estudio de las algas marinas en Chile. Esta reseña recuerda sus inicios, su pionera investigación en botánica y trabajos en microalgas en aguas límnicas, su contribución para el desarrollo de la Estación de Biología Marina de Montemar, Universidad de Chile y también por 20 años en la Universidad de Panamá. El Prof. Llaña estudia, asimismo, las macroalgas marinas robusteciendo la importancia de su valor comercial. En la actualidad, Chile es reconocido como uno de lo más importante proveedores de algas marinas para la extracción de agar, carragenanos y alginatos, esenciales para la industria alimentaria, cosmética, y biomédica.

Palabras clave: Botánico chileno precursor, micro y macroalgas

El 15 de marzo de 1913, hace ciento siete años, nacía en Taltal un niño bautizado como Alfredo Hipólito Llaña Garín, hijo de Rosa Garín Zelada matrona, y de Alberto Llaña Varas, farmacéutico. Hizo sus estudios primarios en su ciudad natal y los secundarios en el Liceo de Antofagasta. Obtuvo su Licencia Secundaria en 1930, matriculándose en 1931 en el Instituto Pedagógico de la Universidad de Chile, en Santiago. Allí obtuvo el Título de Profesor de Ciencias Biológicas y Química, con distinción unánime el 29 de diciembre de 1937.

En el segundo año de su carrera universitaria, fue designado Ayudante de la Cátedra de Botánica del Instituto Pedagógico, cuyo Profesor Titular era Don Víctor Manuel Baeza, destacado discípulo y antiguo ayudante del Prof. Dr. Federico Johow. De nacionalidad alemana, el Dr. Johow, profesor de alemán y sabio naturalista, tuvo una destacada trayectoria en la Facultad de Medicina y en la Facultad de Filosofía y Educación de la Universidad de Chile. Más tarde el Prof. Alfredo Llaña fue Profesor Auxiliar de Botánica en el mismo Instituto Pedagógico (Fig. 1) y al fallecer
Don Víctor Manuel Baeza, lo sucedió en la Cátedra. En los años que trabajaron juntos habían hecho una petición al Decano de la Facultad de Filosofía y Educación, Dr. Yolando Pino Saavedra y fue sobre el deseo de contribuir a la celebración del Centenario de la Universidad de Chile por lo cual necesitaba ayuda económica para efectuar un viaje a la zona austral del país para la búsqueda de material botánico fresco. Por tanto, los profesores Baeza y Llaña logran terminar uno de sus trabajos de investigación en curso, recorriendo las provincias de Arauco, Cautín, Valdivia y Chiloé. Como resultado de esta herborización y la consulta de los herbarios del Museo Nacional de Historia Natural, del Departamento de Genética y Fitotecnia del Ministerio de Agricultura, el Herbario Johow del Instituto Pedagógico y el Herbario particular del Prof. Dr. Karl Grandjot, este último distinguido Profesor de Matemáticas en la Universidad de Chile, quien en sus ratos libres se dedicaba a la Botánica, los proponentes lograron finalizar así una publicación sobre Coníferas (Llaña \& Baeza 1942). 

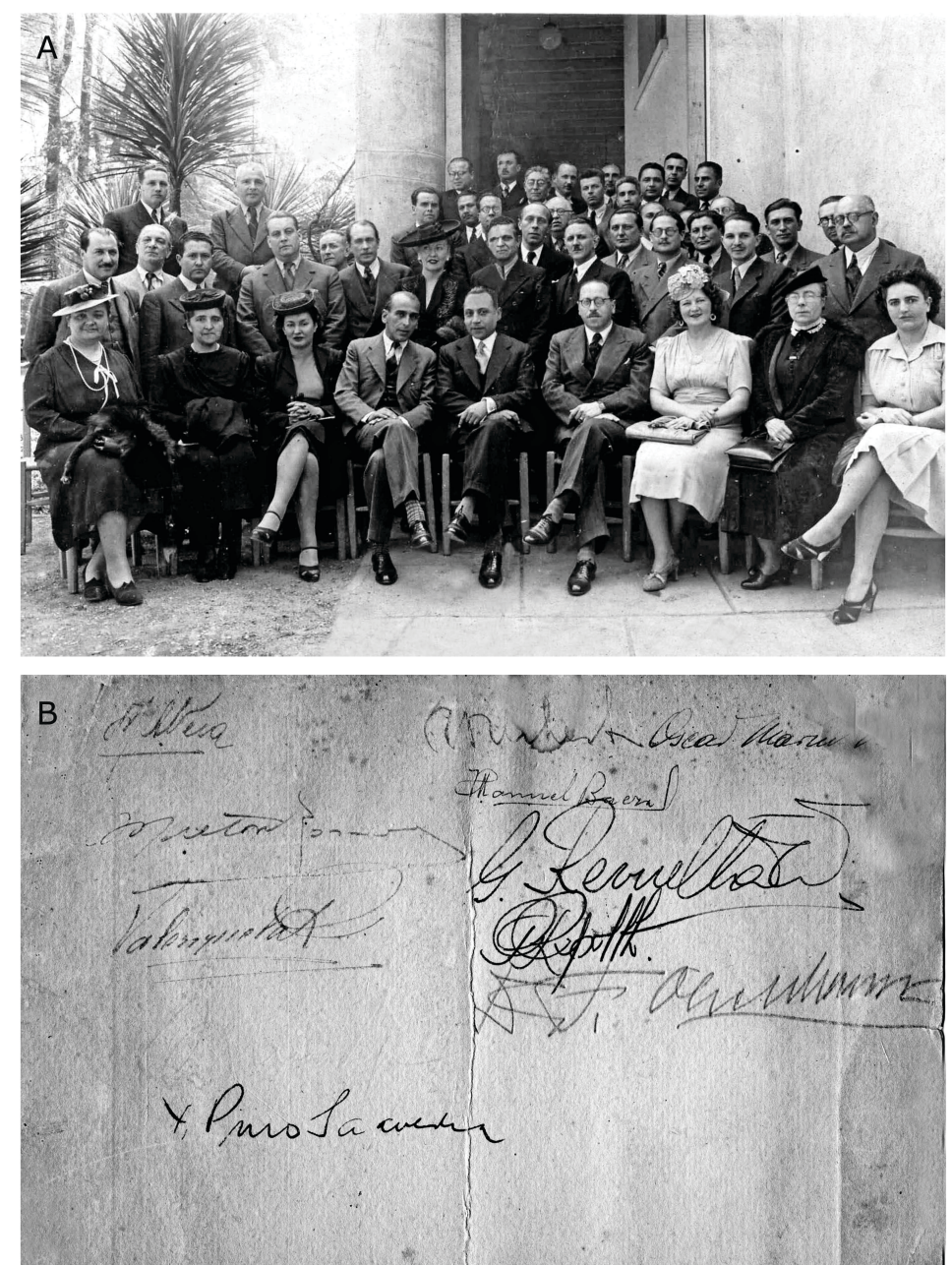

Figura 1. A) Foto circa 1942. Alfredo Llaña (tercero con anteojos segunda fila de derecha izquierda) entre los académicos del Instituto Pedagógico de la Universidad de Chile, Santiago, junto al Dr. Yolando Pino Saavedra, Decano de la Facultad de Filosofía y Educación de la Universidad de Chile y la connotada profesora Irma Salas entre otros. B) Foto al reverso y donde firman Manuel Baeza, Oscar Marín, Fernando Oberhauser, Gumersindo Revuelta, A. Vera y Arturo Valenzuela / A) Photo circa 1942. Alfredo Llaña (third with glasses second row from right left) among the academics of the Instituto Pedagógico, Universidad de Chile, Santiago, together with Dr. Yolando Pino Saavedra, Dean of the Facultad de Filosofía y Educación Universidad de Chile and the renowned teacher Irma Salas among others. B) Photo to the back and signed by Manuel Baeza, Oscar Marín, Fernando Oberhauser, Gumersindo Revuelta, A. Vera and Arturo Valenzuela

Más tarde, Llaña trabaja en diatomeas basado en material obtenido y mantenido en acuarios de su laboratorio y de otros de la Piscicultura de Río Blanco (Llaña 1942a). En este mismo año, termina el manuscrito de su trabajo sobre algunas algas marinas del litoral chileno basado en el material recolectado por los profesores Baeza y Johow, y por él mismo en Coquimbo y Valparaíso. En esta última publicación destaca el valor de las algas chilenas en la alimentación del hombre y de los animales, así como también la posibilidad potencial de aprovechar algunas de ellas en la industria (Llaña 1942b).

Así, poco a poco, el Prof. Llaña se fue especializando en algas, siendo aconsejado y avalado por el Prof. Marcial R. Espínola, a cargo de la Sección Botánica Criptogámica del Museo Nacional. Es por eso que, cuando en julio de 1945 se inauguró la Estación de Biología Marina de la Universidad de Chile en Montemar, Viña del Mar. El Prof.
Llaña junto con los profesores Regina Cubillos y Francisco Riveros, acompañaron como científicos fundadores al Prof. Dr. Parmenio Yáñez y constituyeron así la primera planta profesional: Parmenio Yáñez Andrade (Director), Prof. Alfredo Llaña Garín (Jefe de la Sección Botánica), Prof. Francisco Riveros Zúñiga (Jefe de la Sección Zoología) y Prof. Regina Cubillos Moya (Química).

El Prof. Llaña publica en el primer volumen de la Revista de Biología Marina, dos notas ficológicas sobre Gigartina y Plocaria (Llaña 1948a), una revisión de las algas de Valparaíso (Llaña 1948b) y un visionario trabajo sobre algas marinas industriales, resaltando la importancia de numerosas especies de algas rojas productoras de agar, y algas pardas productoras de algina (Llana 1948c). En este mismo trabajo destaca la importancia del "luche" y "cochayuyo" como importantes componentes de la dieta de poblaciones costeras del centro sur de Chile. 
Actualmente, Chile es uno de los diez países de mayor producción de macroalgas para la extracción de ficocoloides a nivel mundial. Las algas pardas, comúnmente llamados huiros, constituyen la pesquería bentónica de mayor valor social y económico para pescadores artesanales y recolectores de orilla en el norte del país. La extracción de 400.000 ton/año, revelan también su enorme importancia ecológica, puesto que los huiros son organismos ingenieros de ecosistemas y constituyen áreas de refugio, alimentación y reproducción de innumerables invertebrados y peces (Vásquez 2016).

\section{Años Pioneros}

A fines de 1937, Parmenio Yáñez había solicitado al Consejo Universitario de la Universidad de Chile, una comisión de estudios para ir a Europa a perfeccionar sus conocimientos en biología marina. Esta estadía permitiría a su regreso establecer una Estación Biológica dependiente de la Universidad y destinada, no solo a satisfacer las necesidades de docencia e investigación, sino también todas aquellas que la industria pesquera plantease al país. Después de recorrer toda la costa entre Llolleo y Quintero, en el litoral central de Chile, el Dr. Yáñez y sus acompañantes eligieron Montemar, una caleta y un roquerío costero ubicado a 7,2 km al norte de Viña del Mar, Región de Valparaíso. El 17 de abril de 1941 se obtiene la concesión de playa, y el Decreto de Fundación de la Estación de Biología Marina, dictado por la Universidad de Chile el 28 de agosto de 1941. El primer edificio, que contenía dos laboratorios, es inaugurado el 28 de julio de 1945. Por Decreto Supremo del 22 de junio de 1945, se establece que la Estación de Biología Marina trabajaría en estrecha colaboración con el Museo Nacional de Historia Natural de Santiago de Chile y además, desarrollaría una efectiva colaboración con la Sociedad Científica de Chile.

Entre enero y febrero de 1943, Nibaldo Bahamonde lo acompaña en una sacrificada expedición a los bosques de cipreses y alerzales de Piuchué, en las cercanías de Cucao (Isla Grande de Chiloé), en busca de semillas de alerce que le había solicitado el Real Jardín Botánico de Kew. "Alli fue donde me fue posible aquilatar la paciencia, esfuerzo y bonhomía de este investigador". El Prof. Llaña aprovechó el viaje para recolectar algas, y reconoció de paso la presencia de Chondrus sp. en las vecindades de Chonchi, sobre la cual la Compañía de Cervecerías Unidas le había solicitado información ya que deseaba obtener, "agar-agar" en Chile. Este ficocoloide que se utilizaba en el proceso de clarificación de la cerveza, no estaba disponible en el país, ya que la importación se encontraba suspendida por la Segunda Guerra Mundial. "Más tarde se encontrarían en la playa de Quetalmahue, cerca de Ancud otras algas, de los géneros Gelidium y Gracilaria, que nos sirvieron para iniciar un trabajo sobre agarófitas en 1945". Este hallazgo le permitió a Nibaldo Bahamonde el estudio sobre la "Extracción de agar-agar de algas marinas de Chile", y obtener el Grado de Licenciado en Filosofía, con mención en Ciencias Biológicas y Química en la Facultad de Filosofía y Educación de la Universidad de Chile.

Entre febrero y marzo de 1947, se efectuó la Primera Expedición Antártica Chilena y la Estación de Biología Marina estuvo representada por tres miembros de su personal: Parmenio Yáñez, Juan Lengerich y Pedro Brandt. Ellos obtuvieron muestras entre el Estrecho de Magallanes y Bahía Margarita $\left(52^{\circ} 10^{\prime}\right.$ a $68^{\circ} 15^{\prime}$ S $)$ utilizando una pequeña rastra a profundidades no mayores a 20 metros. El material algológico obtenido, fue estudiado por el Prof. Llaña quien publicó sus resultados en la naciente Revista de Biología Marina (Llaña 1948d).

El Prof. Llaña estudió también la "tierra de infusorios", "tiza" o "kieselguhr" de Chiloé, de la cual reconoció y describió su estructura algológica, lo cual le permitió ilustrar los trabajos prácticos de su cátedra. Más aun, con otros aportes contribuyó a modernizar la enseñanza de la Botánica en la Facultad de Filosofía y Educación de la Universidad de Chile, iniciando los estudios de histología, organología y fisiología vegetal. En su trayectoria fue el que profundizó la enseñanza de la Botánica Criptogámica en el Instituto Pedagógico, especialmente el de las algas.

\section{TRAYECTORIA}

Alfredo Hipólito Llaña Garín (Fig. 2), es sin duda el precursor de la ficología marina en Chile. Siendo fundador y jefe de la Sección Botánica de la Estación de Biología Marina de Montemar, es nombrado por Decreto $N^{\circ} 1245$ del 8 de julio de 1948 para formar parte de la comisión organizadora del Primer Congreso Latino Americano de Oceanografía, Biología Marina y Pesca, el cual se celebraría entre el 5 y 10 de septiembre de 1949 en las ciudades de Valparaíso y Viña del Mar. En dicho Congreso, el Prof. Llaña asume la presidencia de la subcomisión sobre Botánica Litoral y Marina.

De forma pionera inició la algoteca de la Estación de Biología Marina de Montemar (hoy Facultad de Ciencias del Mar y de Recursos Naturales de la Universidad de Valparaíso) y publicó los primeros resultados de trabajos algológicos marinos hechos por chilenos (Llaña 1942, 1948). Con anterioridad solo había información publicada sobre algas límnicas por el Prof. Marcial Espinosa (1923), del Museo Nacional de Historia Natural, el cual fue otro de los discípulos distinguidos del Dr. Federico Johow. 


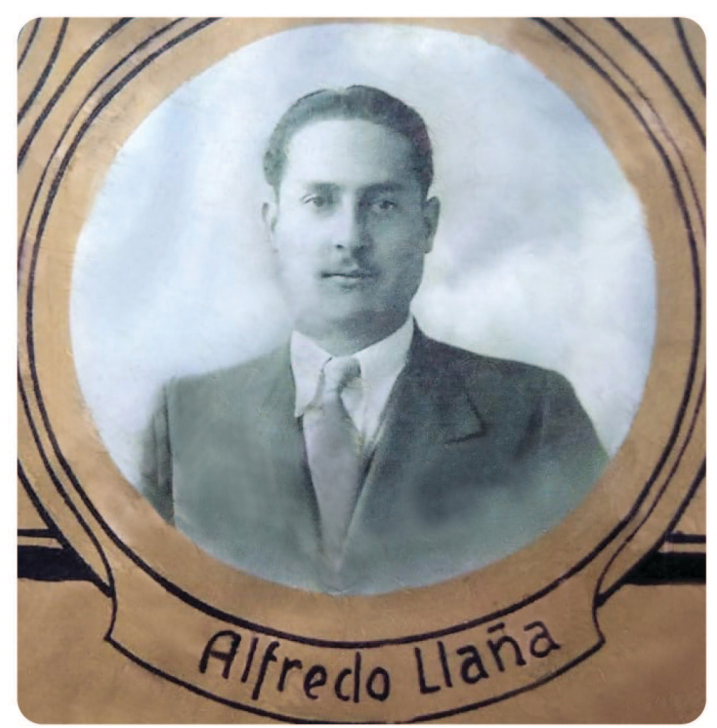

Figura 2. Retrato fotográfico del Prof. Alfredo Hipólito Llaña Garín / Photographic portrait of Prof. Alfredo Hipólito Llaña Garín

Posteriormente, es mandatado por el Honorable Consejo Universitario de la Universidad de Chile, para realizar estudios ficológicos en las costas del Pacífico sudamericano. En 1945, obtuvo una beca de la Fundación Rockefeller para estudiar botánica, especialmente algas, en Ann Harbour, Universidad de Michigan, bajo la dirección del Dr. William Taylor, donde obtuvo un Master en Ciencias y le conservaron la beca para doctorarse. Viajó por varios laboratorios de Biología Marina de los Estados Unidos de Norteamérica (EEUU), incluyendo el Woods Hole Oceanographic Institute, el laboratorio marino John Hopkins (Hopkins Marine Station) y el Scripps Institution of Oceanography de la Universidad de California, en La Jolla, San Diego, California. Estuvo un mes en el New York Botanical Garden para estudiar la organización y mantención del Herbario. Visitó las Universidades de Harvard, de Yale, New York, California, Chicago e Iowa, y algunos otros laboratorios de Canadá para conocer los métodos de trabajo y posibilidades industriales con las algas. Además recolectó material algológico en la costa Este y SE de los EEUU para incrementar el Herbario de la Estación de Biología Marina de Montemar.

En 1946, mientras trabajaba en el laboratorio de Botánica de la Universidad de Michigan, se encargó a Pedro Brandt, patrón de pesca de la Estación de Biología Marina de Montemar para recolectar algas marinas en Arica, Iquique, Antofagasta y Coquimbo y ser enviadas al Prof. Llaña para su estudio. Permaneció en América del Norte hasta 1947. A principios de junio de 1948, fue comisionado por la Universidad de Chile para realizar un viaje científico a lo largo del Pacífico Sur y Centroamérica para estudiar las algas de valor económico y recolectar durante tres meses, material para incrementar el Herbario Ficológico de la Estación de Montemar.
Según Rivera (2006), los primeros artículos sobre diatomeas escritos por investigadores chilenos, aparecen durante la segunda década del siglo XX, tales como los de Espinosa (1917) quien publicó sobre las diatomeas provenientes de Piuchué, y el de Espinosa (1923) que publicó una lista sistemática de algunas algas chilenas de agua dulce. Transcurren dos décadas hasta que Llaña (1942a), publicara los primeros antecedentes sobre las especies fitoplanctónicas que habitan nuestros ríos, lagos lagunas y estuarios, estudiados hasta entonces sólo por expediciones extranjeras (Montecino 1985).

Más tarde concurrió como profesor invitado a Costa Rica y a otros países de Centroamérica, prosiguiendo sus investigaciones botánicas. Trabajos suyos fueron enviados a España para su publicación (no fue posible conseguir datos sobre ellos). Como en mayo de 1950, el Prof. Llaña, aún no regresaba a Montemar y no se tenían noticias de él, el 1 de junio de ese año se declaró vacante su cargo y se nombró, como Jefe de la Sección Botánica de la Estación de Biología Marina, al Profesor Héctor Etcheverry Daza, quién desde 1948 trabajaba ya en calidad de huésped científico, en reemplazo del Prof. Llaña.

Es interesante recordar que, además de sus actividades científicas, Alfredo Llaña participaba también en grupos literarios. Fue amigo de escritores como Juan Godoy y Mariano Latorre (Director del Instituto Pedagógico de Santiago en 1945), con quien compartió datos botánicos sobre su libro "Zurzulita". También hacía caricaturas y escribía cuentos. Entre ellos: "El Cachimba" (Llaña 1941) que aparece en una Antología de cuentos chilenos recopilados por Nicomedes Guzmán, quien al presentarlo hace un elogioso comentario sobre el autor. Guzmán (1941) escribe "Marín" por "Garín" equivocando el segundo apellido lo que dificulta conocer esta veta literaria, con mucho de social e "Imaginismo" de Alfredo Llaña.

Durante su estadía en Panamá por más de 20 años, contribuyó a organizar la Facultad de Ciencias Naturales y Farmacéuticas de la Universidad de Panamá, e incluso tuvo la oportunidad de contribuir al diseño del edificio. En representación de esa Facultad inició los primeros contactos y participó en las primeras reuniones con las autoridades respectivas para sentar las bases de la Facultad de Agronomía, cuya Escuela de Agronomía, en un comienzo, formó parte de la Facultad de Ciencias Naturales y Farmacéuticas. Allí, junto al Prof. panameño Alejandro Méndez Pereira, son recordados por sus discípulos como dos grandes "colosos" de la botánica, ellos ayudaron a establecer esta disciplina en la Escuela de Biología y "se dedicaron casi con exclusividad a la docencia universitaria y a la organización de la incipiente Facultad de Ciencias. También contribuyeron a organizar el Herbario de la Universidad de Panamá. 
Don Alfredo Llaña contrajo matrimonio en abril de 1937 con la Prof. Elena Mena Vergara, quien fue ayudante del Prof. Fernando Oberhauser en el Departamento de Química del Instituto Pedagógico de la Universidad de Chile, con quien tuvo 2 hijos. Mónica Elena, Profesora del Departamento de Educación de la Facultad de Ciencias Sociales de la Universidad de Chile y Alfredo Alberto, Arquitecto. Durante su residencia en Centro América dejó una descendencia de 12 hijos. Lamentablemente, falleció a la edad de 57 años, accidentalmente en Panamá el 25 de febrero de 1971.

En el 2014, la Sociedad Chilena de Ficología en el marco del IX Congreso Nacional de Micro y Macroalgas, desarrollado en Viña del Mar, instauró el Premio “Alfredo Llaña Garín”, para distinguir, a quienes han realizado destacados aportes a la ciencia, en particular a la Ficología y en la formación de jóvenes científicos (Tabla 1). Esta distinción se entrega de acuerdo con los respectivos Congresos, cada tres años.

\section{Agradecimientos}

Los autores dejan constancia que Eugenia Navas y Nibaldo Bahamonde fueron los que escribieron originalmente esta reseña. En su momento agradecieron a su esposa Elena Mena, por su ayuda en la recopilación de algunos datos biográficos y fotografías (Fig. 1). Agradecemos a su hija Mónica Elena, por información adicional y la fotografía de su padre (Fig. 2) restaurada por Yanira Tala.

\section{LITERATURA CITADA}

Espinosa M. 1917. Los alerzales de Piuchué - Bacillarieae. Boletín Museo Nacional de Historia Natural, Chile 10: 82-83.

Espinosa M. 1923. Lista sistemática de algunas algas chilenas de agua dulce. Revista Chilena de Historia Natural 27: 93-96.

Llaña AH. 1941. Cachimba. En: Guzmán N (ed). Nuevos cuentistas chilenos, pp. 167-180. Editorial Cultura, Santiago de Chile.
Tabla 1. Algólogos distinguidos por la Sociedad de Ficología de Chile con el Premio "Alfredo H. Llaña Garín" / Algologists distinguished by the Sociedad de Ficología de Chile with the "Alfredo H. Llaña Garín" Award

\begin{tabular}{lll}
\hline Año & Ficólogo premiado & \multicolumn{1}{c}{ Afiliación } \\
\hline \multirow{2}{*}{2014} & Krisler Alvear & Universidad de Concepción \\
& María Eliana Ramírez & Museo Nacional de Historia Natural \\
& Bernabé Satelices & Pontificia Universidad Católica de Chile \\
2017 & Erika Fonck & Universidad Católica del Norte \\
& Mariela González & Universidad de Concepción \\
2020 & Vivian Montecino & Universidad de Chile \\
& Julio A. Vásquez & Universidad Católica del Norte \\
\hline
\end{tabular}

Llaña AH. 1942a. Contribución al estudio de las diatomeas chilenas. Anales de la Facultad de Filosofía y Educación. Sección Biología, Cuaderno 1: 41-51.

Llaña AH. 1942b. Algunas algas del litoral chileno. Anales de la Facultad de Filosofía y Educación, Sección Biología, Cuaderno 1: 53-61.

Llaña AH. 1948a. Dos notas ficológicas: Gigartina chondrioides Bory y Plocaria chondroides Montagne y Thorea chilensis Montagne. Revista de Biología Marina 1: 59-61.

Llaña AH. 1948b. Algas marinas de Valparaíso. Revista de Biología Marina 1: 81-90.

Llaña AH. 1948c. Algas industriales de Chile. Revista de Biología Marina 1: 124-131.

Llaña AH. 1948d. Primera expedición Antártica chilena. Algas Marinas. Revista de Biología Marina 1(1): 19-31.

Llaña AH \& VM Baeza. 1942. Clase Coníferas. Anales de la Facultad de Filosofía y Educación, Sección Biología, Cuaderno 1: 5-28.

Montecino V. 1985. Fitoplancton estuarial y de aguas límnicas. Revista Biología Marina 21: 199-211.

Rivera P. 2006. Estado de conocimiento de las diatomeas dulceacuícolas de Chile. Gayana 70(1): 1-7.

Vásquez JA. 2016. The fishery of brown seaweed in Chile. In: Mikkola $\mathrm{H}$ (ed). Fisheries and aquaculture in the modern world, pp. 123-141. In Tech, Rijeka. 